\title{
Biological importance of sciadopitysin on hepatic and renal toxicity: biological role in the medicine
}

\author{
Dinesh Kumar Patel
}

Faculty of Health Sciences, Sam Higginbottom University of Agriculture, Technology and Sciences, Payagraj, India

Background: More than 122 compounds used in the medicine for the treatment of human disorders were derived from plant sources. Nowadays, herbal based medicine in therapies has become popular which signified the rapidly increasing worldwide consumption of herbal drug and their derived herbal medicines.

Methods: Scientific research work revealed the presence of sciadopitysin in different medicinal plants of potential health benefit. Numerous scientific research work data has been collected in the present work and analyzed to know the health beneficial aspects of sciadopitysin in the medicine. Biological importance of sciadopitysin on hepatic and renal toxicity has been investigated through literature data analysis of various scientific research works.

Results: Literature data analysis revealed the biological importance of sciadopitysin in the medicine. Literature data analysis revealed that sciadopitysin reduced cell viability which signified the biological importance of sciadopitysin on hepatic and renal toxicity. Literature data analysis revealed acute renal failure induced by sciadopitysin in the literature. Other pharmacological data analysis signified the biological importance of sciadopitysin in the medicine.

Conclusions: Literature data analysis revealed the biological importance of sciadopitysin on Hepatic and renal toxicity.

Corresponding author: Dinesh Kumar Patel

E-mail:dkp.itbhu@gmail.com

(c) The Korean Society for Transplantation

This is an Open Access article distributed under the terms of the Creative Commons Attribution Non-Commercial License (http://creativecommons.org/licenses/by-nc/4.0/) which permits unrestricted non-commercial use, distribution, and reproduction in any medium, provided the original work is properly cited. 